\title{
GESTÃO ESTRATÉGICA E TURISMO SUSTENTÁVEL: UM ESTUDO BIBLIOMÉTRICO DA PRODUÇÃO CIENTIFFICA NA BASE WEB OF SCIENCE NO PERIODO DE 2007 A 2017
}

LUCIANA APARECIDA BARBIERI DA ROSA

Doutoranda em Administração de Empresas, Universidade Federal de Santa Maria - UFSM, Brasil. lucianaaparecidabarbieri@yahoo.com.br

CAROLINE ROSSETO CAMARGo

Mestre em Administração, Universidade Federal de Santa Maria - UFSM, Brasil.

thiagoc@utfpr.edu.br

Maria CARolina Martins RODRIGUES

Doutora em Administração. Universidade de Extremadura - UEX, Espanha.

rodriguescarolina@live.com.pt

CLANDIA MAFFINI GOMES

Doutora em Administração, Universidade de São Paulo - FEA/USP, Brasil.

Professora do Departamento de Ciências Administrativas da Universidade Federal de Santa Maria - UFSM, Brasil.

clandiamg@gmail.com

\section{Resumo}

Este artigo teve como propósito apresentar o Estado da arte e procurar discutir as interfaces e articulações entre os constructos Cestão Estratégica e Turismo Sustentável. Dessa forma, o objetivo deste artigo é analisar as características das publicações relacionadas com o tema Gestão Estratégica e Turismo Sustentável, no período de 2007 a 2017. A metodologia utilizada foi uma pesquisa bibliométrica, descritiva. A pesquisa foi realizada através da base de dados Web of Science da ISI Web of Knowledge, totalizando 176 artigos selecionados. Os resultados da pesquisa mostram que o ano de maior número de publicações foi 2017 com 37. Journal of Sustainable Tourism apresentou o maior número de publicações com o total de 14.

Palavras-chave: bibliometria; gestão estratégica; turismo sustentável. 


\title{
STRATEGIC MANAGEMENT AND SUSTAINABLE TOURISM: A BIBLIOMETRIC STUDY OF SCIENTIFIC PRODUCTION ON THE WEB OF SCIENCE IN THE 2007-2017 PERIOD
}

\begin{abstract}
This article had as its purpose to present the State of the art and to try to discuss the interfaces and articulations between the constructs Strategic Management and Sustainable Tourism. Thus, the objective of this paper is to analyze the characteristics of the publications related to the theme Strategic Management and Sustainable Tourism, from 2007 to 2017. The used methodology was a bibliometric, descriptive research. The research was conducted through the Web of Science database of ISI Web of Knowledge, totaling 176 selected papers. The research's results show that the year with the greatest number of publications was 2017 (37). The Journal of Sustainable Tourism presented the largest number of publications with a total of 14.
\end{abstract}

Keywords: bibliometry; strategic management; sustainable tourism.

\section{GESTIÓN ESTRATÉCICA Y TURISMO SUSTENTABLE: UN ESTUDIO BIBLIOMÉTRICO DE LA PRODUCCIÓN CIENTIFICA EN LA WEB DE LA CIENCIA EN EL PERIODO 2007-2017}

\begin{abstract}
Resumen
Este artículo tenía como propósito presentar el estado del arte y tratar de discutir las interfaces y articulaciones entre los constructos Gestión Estratégica y Turismo Sustentable. De esta forma, el objetivo de este artículo es analizar las características de las publicaciones relacionadas con el tema Cestión estratégica y turismo sustentable, del periodo 2007 al 2017. La metodología utilizada fue una investigación bibliométrica y descriptiva. La investigación se realizó a través de la base de datos de Web of Science de ISI Web of Knowledge, totalizando 176 artículos seleccionados. Los resultados de la investigación muestran que el año con el mayor número de publicaciones fue 2017 (37). Journal of Sustainable Tourism presentó el mayor número de publicaciones con un total de 14.
\end{abstract}

Palabras clave: bibliometría; gestión estratégica; turismo sustentable. 


\title{
INTRODUÇÃO
}

As empresas prestadoras de serviço, para serem cada vez mais competitivas devem procurar entender a importância de implementação de estratégia em suas gestões (Pontes, 2012). Dessa forma, o planejamento estratégico necessita ser simplificado sendo primordial o engajamento e comprometimento de toda a equipe (Almeida, 2010).

A estratégia empresarial equipara-se aos objetivos delineados pela direção com o intuito de atingir os resultados aspirados pela empresa futuramente. Dessa maneira, é essencial que a organização possua propósitos bem delineados com a finalidade de desenvolver métodos que tragam resultados para a organização (Mintzberg, Lampel, Quinn \& Ghoshal, 2006). À vista disso, de acordo com Martins, Filho e Nagano (2015), a administração de boas práticas de gestão são essenciais, embora, diversas circunstâncias apresentem um incentivo para qualquer empresa, especialmente às de pequeno porte.

Neste sentido, a origem de novas estratégias que rejeitem estímulos presentes e futuros, e as transmutações nos empreendimentos turísticos, requerem políticas eficientes de repontarem as questões tais como reorganização econômica, social em pontos rurais e urbanos (Balsadi, 2001), assim como em países que aspiram amplificar o turismo com propósitos de gerar investimento, criando trabalho e desenvolvimento da localidade.

Visto a importância da elaboração de estratégias para os empreendimentos turísticos, outra variável eminente é a sustentabilidade que pode ser compreendida, consoante a ótica da Organização Mundial do Turismo (OMT), como aquela que deve "satisfazer as necessidades presentes sem comprometer a possibilidade de gerações futuras" e que "permite o desenvolvimento sem degradar ou esgotar os recursos que tornam possiveis o mesmo desenvolvimento" (OMT, 2001. p. 245).

Logo, alguns objetivos podem ser delineados para que o turismo sustentável possa auxiliar no desenvolvimento económico:

\begin{abstract}
"melhorar a qualidade de vida da população local, das pessoas que vivem e trabalham no local turístico; prover experiência de melhor qualidade para o visitante; manter a qualidade do meio ambiente da qual depende a população local e os visitantes; a efetivação de aumento dos níveis de rentabilidade econômica da atividade turística para os residentes locais; assegurar a obtenção de lucros pelos empresários turísticos." (OMT, 2001. p. 246).
\end{abstract}

Com isso, o objetivo do artigo é apresentar o estado da arte e procurar discutir as interfaces e articulações entre os construtos Cestão Estratégica e Turismo Sustentável. Após a breve introdução, serão apresentados os principais temas desta pesquisa: a seção de revisão de literatura, logo após os procedimentos metodológicos da pesquisa e, na seção seguinte, os principais resultados encontrados. Para concluir o estudo, são realizadas as considerações finais, bem como as suas limitações e futuras linhas de investigação.

\section{REVISÃO DE LITERATURA}

\section{Gestão estratégica Sustentabilidade}

O conceito de estratégia nos negócios surgiu a partir da década de 50, quando as empresas sentiram a necessidade se preparar para o futuro, pois a sobrevivência não era mais garantida com a delimitação de objetivos e ações de curto prazo. Era preciso criar visão de grande escopo e de longo prazo (Motta, 1995). 
Pode-se descrever a estratégia como sendo, um conjunto coordenado e integrado de ações e compromissos, definido para explorar capacidades fundamentais e obter vantagem competitiva, definição utilizada tanto para propósitos de organização como de ambientes (Hitt, Ireland \& Hoskisson, 2015). As estratégias devem estar alinhadas com a inovação, além de ser fundamental estarem coordenadas com as estratégias da empresa (Wakeford et al., 2017). Parnell (2010) complementa que cada empresa possui sua própria estratégia, no entanto, é possível identificar grupos que executam métodos genéricos semelhantes associados com a competitividade dos negócios (Kneipp, 2016).

Nicolau (2001) argumenta que as conceptualizações de estratégias são tão numerosas quanto os autores que as referem, existindo, porém, diferenças em alguns aspectos, tais como a base do conceito, o conteúdo e os processos de formação da estratégia. Para os autores Hamel (2001) e Farjoun (2002), estratégia é como um plano ou modelo que une as metas, as políticas e as sequências de ações principais de uma organização em um todo coeso. Conforme Diniz, Giarola, Balbino, Ferreira e Nazareth (2013) as empresas tendem a estabelecer estratégias que atinjam ao maior os resultados condizentes com os objetivos traçados.

Desta maneira, alguns estudos empíricos descrevem que as empresas brasileiras estão gerenciando suas estratégias voltadas para estratégias inovadoras, assim contribuindo para a inserção dos sistemas (Hall \& Matos, 2010). Como visto, os conceitos do termo estratégia podem ser resumidamente definidos como a orientação e a finalidade de uma empresa para se manter competitiva em mercados turbulentos.

Para Porter (2005), a estratégia competitiva tem por finalidade estabelecer uma posição sustentável e lucrativa em oposição as forças que motivam a competição entre empresas. E, essas distintas concepções e abordagens à temática estratégica podem ser empregues em distintos métodos (Oliveira, 2013).

Nesta mesma ótica, as transformações tecnológicas, sociais, culturais que acontecem na aldeia global fazem com que as empresas prestadoras de serviços busquem novas formas de crescimento, desenvolvimento ou aprimoramento para a competitividade empresarial. Isto torna-se substancial na investigação de dados sólidos que evidenciem através de experiências a importância da Gestão Estratégica (Pontes, 2012).

Contudo, compreender os fatores que constituem o micro e macro ambiente das empresas, elaborar o processo de planejamento e deste modo transformar em processos estratégicos que direcionam aos objetivos estabelecidos, devem incluir a preocupação com o meio ambiente e a responsabilidade social, para que se possam identificar oportunidades e definir a prioridade dos investimentos nestas áreas e, simultaneamente, relacioná-las com o pilar econômico (Freire, 2008; Lima e Luz., 2012).

Assim, o Planejamento Estratégico possibilita analisar a posição das empresas em relação aos três pilares da sustentabilidade, organizando-as para atuar no mercado e estabelecer prioridades para 0 seu desenvolvimento sustentável da região ou país onde atua. (Dias, 2006; Savitz e Weber, 2007). No próximo tópico será explanado sobre o turismo sustentável.

\section{TURISMO SUSTENTÁVEL}

De acordo com a Organização das Nações Unidas para a Educação, a Ciência e a Cultura (UNESCO), a Organização das Nações Unidas (ONU) proclamou 2017 como o ano internacional do turismo sustentável para o desenvolvimento, como forma de reconhecimento sobre a importância do turismo para a economia. Essa indústria de serviços movimenta cerca de $10 \%$ da economia mundial, contribuindo com a diminuição da pobreza e o aumento do diálogo intercultural. 
Conforme a Organização Mundial do Turismo (OMT, 2014, p. 3), "O turismo compreende as atividades que realizam as pessoas durante suas viagens e estadias em lugares diferentes ao seu entorno habitual, por um período consecutivo inferior a um ano, com a finalidade de lazer, negócio ou outras".

Segundo Araújo, Temoteo, Andrade \& Trevizan (2017), o turismo representa 5\% do PIB global; 235 milhões de empregos em todo o mundo, sendo um dos setores em que o crescimento econômico do mundo é o mais célere. Em 2030, espera-se a visita de 1,8 bilhão de turistas internacionais e, US\$ 1,03 bilhões de divisas gerados pelos turistas.

A inserção do turismo tem ocasionado a substituição de atividades tradicionais, como os pescadores artesanais, cujos filhos abandonam o trabalho dos pais e se tornam funcionários nos meios de hospedagem e de pequenos agricultores que perdem o interesse pelo trabalho sacrificante nas lavouras para trabalharem como guias de trilhas ecológicas (Lozada, 2017).

A World Travel e Tourism Council (WTTC, 2012) define turismo sustentável como ecologicamente suportável em longo prazo, viável economicamente e socialmente responsável para as comunidades e seus moradores através da integração do ambiente natural, humano e cultural. 0 turismo sustentável inclui vários segmentos e nichos, principalmente o ecoturismo e o turismo de natureza, ambos revelado uma mudança profunda da mentalidade dos turistas. Essa nova forma de viajar promove a redução do uso dos transportes individuais e uma melhor distribuiç̧ão regional dos fluxos turísticos.

Para Liu, Vogt, Luo, He, Frank, \& Liu (2012) envolver comunidades locais na conservação e usar ferramentas de mercado para agregar valor económico à biodiversidade, são abordagens que fornecem fontes alternativas de renda para as comunidades locais, ajudando a reduzir a pobreza. Atitudes de conservação e aconselhamentos ambientais podem alterar comportamentos não sustentáveis de extração de recursos e reduzir a pressão humana sobre os sistemas naturais.

Em consonância, Medeiros \& Moraes (2013) afirmam que o envolvimento da população local é essencial para obter sucesso em um desenvolvimento sustentável a longo prazo. A melhor qualidade de vida alcançada pelos anfitriões estabelece uma relação harmoniosa entre turistas e comunidade. A inclusão social e o aumento da renda proporcionam um desenvolvimento integrado baseado na conservação do meio ambiente e da comunidade.

A sustentabilidade surge também como um diferencial competitivo para as empresas do setor turístico, que contribuem para o desenvolvimento socioambiental e econômico das comunidades do entorno ao promoverem o turismo sustentável. Para Pires (2002), qualquer atividade turística deve ser socialmente responsável. O autor cita como princípio universal da sustentabilidade a necessidade de preservar os recursos para que as futuras gerações possam usufruir da mesma forma que as gerações atuais, salientando que a definição de sustentabilidade baseia-se nesse princípio.

O conceito de turismo sustentável considera os três pilares da sustentabilidade, isto é, crescimento econômico, sustentabilidade ambiental e equidade social (Rocha e Zouain, 2015). 0 turismo sustentável foi definido a partir da identificação da necessidade de desenvolver turismo no nível local. Baseado nisso, alguns indicadores para monitorar o turismo sustentável tem emergido: caracterizar o estado atual das coisas, os fenômenos e analisar seu desenvolvimento e mudanças. A utilização de indicadores de sustentabilidade pode ser considerada uma grande vantagem. Os Indicadores de sustentabilidade do turismo, especificamente, provém informações importantes necessárias para entender melhor os problemas econômicos, sociais e ambientais, criando e conduzindo políticas e projetos de desenvolvimento (Butler, 2004).

Leví (2012) cita a Agenda 21 para a indústria de Viagens e Turismo, documento publicado em 1993 pela Organização Mundial do Turismo (OMT), pelo World Travel \& Tourism Council (WTTC) e pelo Conselho da Terra como definidores do conceito do desenvolvimento sustentável. O documento 
também define áreas prioritárias de ação e enfatiza o poder das parcerias entre governos, agentes de turismo e organizações não-governamentais, além de citar vantagens de transformar o turismo em uma atividade sustentável.

A operacionalização do turismo sustentável é ainda bastante complexa, apesar de todos os instrumentos criados para colocar em prática todos os conceitos. Assim, o turismo sustentável precisa adotar um planeamento adequado, que permita acrescentar valor aos destinos, ao mesmo tempo que trazer benefícios para os diversos atores envolvidos e minimiza os efeitos negativos (Leví, 2012).

\section{METODOLOGIA}

O presente estudo bibliométrico, caracteriza-se quanto à abordagem como quantitativa descritiva, com o objetivo de investigar as principais utilizações do tema Gestão Estratégica e Turismo Sustentável (Strategic Management and Sustainable Tourism) nos debates teóricos e empíricos, no período de 2007 a 2017.

Para a coleta de dados desta pesquisa utilizou-se a base de dados Web of Science, pois, trata-se de uma das maiores bases multidisciplinares que englobam dados, resumos e citações da literatura científica. Nos campos de pesquisa colocou-se as palavras Gestão Estratégica e Turismo Sustentável, e se identificou os principais autores que escreveram sobre o tema, dentre outras características. Investigaram-se artigos, publicados nesse período, com a finalidade de mostrar 0 contexto em que o termo é referenciado.

\section{DEFINIÇÃO DO ESCOPO DO ESTUDO}

A investigação das publicações para a realização da bibliometria realizou-se a partir dos mecanismos de pesquisa da base de dados da Web of Science (WOS) do Institute for Scientific Information (ISI). A WOS consiste numa base multidisciplinar que detém aproximadamente 12.000 jornais indexados. A base indexa apenas os jornais mais citados nas suas respetivas áreas, como por exemplo: Journal of Sustainable Tourism, Tourism Management, Procedia Social and Behavioral Sciences, Sustainability, International Multidisciplinary Scientific Geoconference Sgem, WIT Transactions on Ecology and The Environment e AEBMR Advances In Economics Business and Management Research.

A WOS é também um índice de citações, referindo, para cada artigo, os documentos por ele citados e os documentos que o citaram (Gráfico 1). 
GRÁFICO 1- TIPO DE DOCUMENTOS

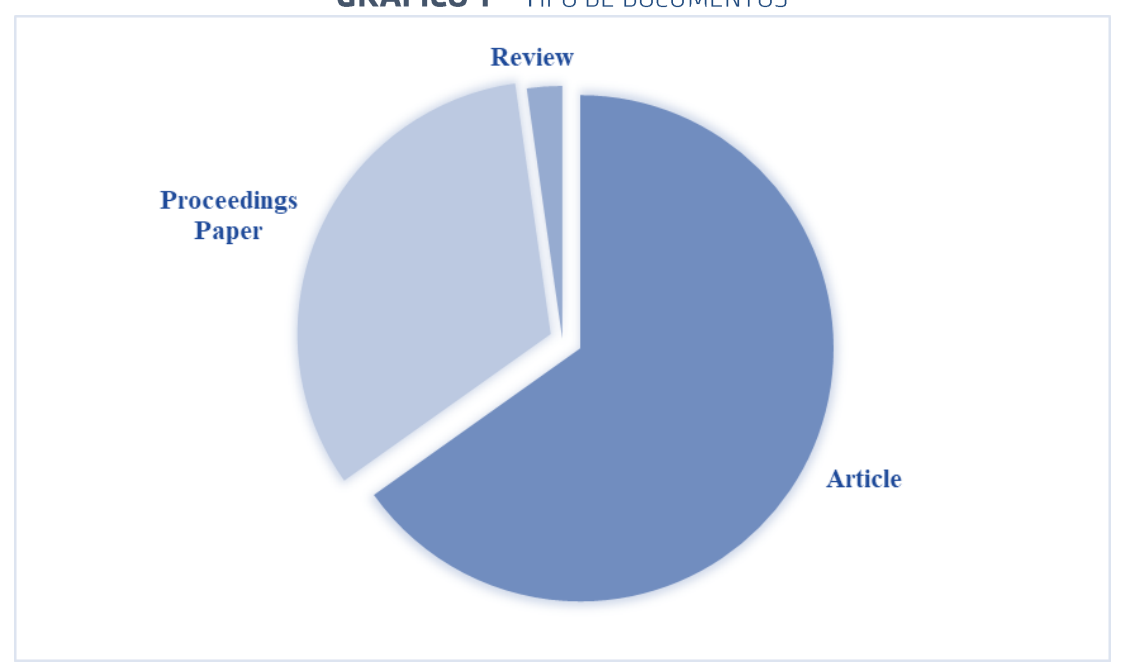

Fonte: Elaborado pelas autoras.

A bibliometria é uma técnica estatística ou matemática que analisa as características e desenvolvimento de diversas áreas científicas e o cálculo de índices de citações (Okubo, 1997; Araújo, 2006 e Rostaing, 1997). Para Silva (2004), a bibliometria tem como objetivo analisar a atividade científica através do estudo quantitativo das publicações.

Foram pesquisados os termos Strategic Management and Sustainable Tourism na Web of Science no período compreendido entre os anos de 2007 e 2017, resultando um total de 176, sendo 116 (cento e dezasseis) artigos, 58 (cinquenta e oito) proceedings.

$\mathrm{Na}$ análise bibliométrica do presente estudo procurou-se identificar as variáveis seguintes: principais autores, título das fontes, principais instituições, ano das publicações, principais países e idiomas, áreas do conhecimento e a relação entre autores com mais publicações e as mais citadas.

\section{ETAPAS DA COLETA DOS DADOS}

Esta pesquisa dividiu-se em duas etapas. Inicialmente digitaram-se os termos Strategic Management and Sustainable Tourism no campo de pesquisa da base WOS, delimitando-se ao período entre 2007 a 2017. Em seguida procedeu-se ao levantamento das características gerais das publicações.

Na segunda etapa foram comparadas as publicações mais citadas com os autores que mais publicaram no mesmo período. A Figura 1 evidência as etapas da pesquisa. 


\section{FIGURA 1 - ETAPAS DA PESQUISA}

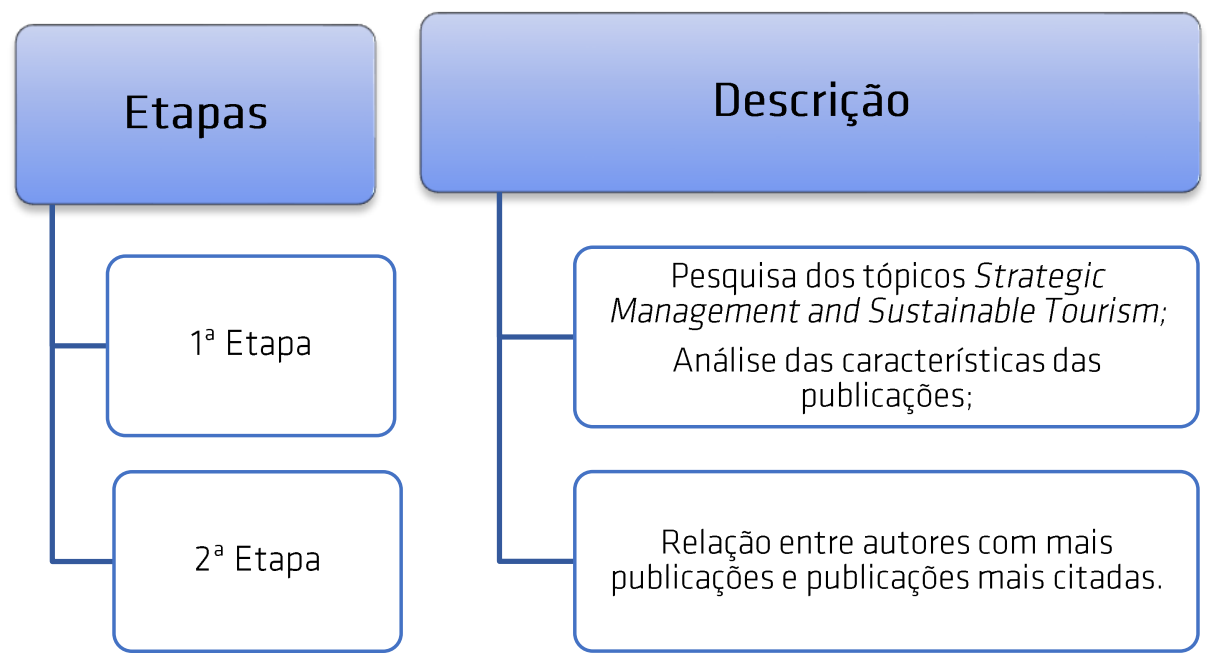

Fonte: Elaborado pelas autoras.

Assim, de acordo com as etapas descritas na Figura 1 foi realizada a análise bibliométrica do referido estudo, apresentada a seguir.

\section{ANÁLISE E DISCUSSÃO DOS RESULTADOS}

Os resultados da pesquisa apresentados a seguir identificam as principais características da produção científica na base de dados da Web of Science relacionada às palavras-chave Gestão Estratégica e Turismo Sustentável no período 2007 a 2017. Depois de refinar a pesquisa para as categorias de Gestão Estratégica e Turismo Sustentável e optar por apresentar resultados referentes apenas a artigos, obteve-se o total de 176 artigos. Em primeiro lugar serão apresentadas as características gerais das publicações: principais autores, título das fontes, principais instituições, ano das publicações, principais países e idiomas e áreas do conhecimento. Por último, serão apresentados o número de publicações por autor e o número de citações.

\section{CaRActeristicas Gerais das publicações sobre Strategic Management and Sustainable Tourism: na WEB OF SCIENCE}

Apresentam-se a seguir, as características gerais das publicações relacionadas com os temas Strategic Management and Sustainable Tourism nas seguintes categorias: principais autores, título das fontes, instituições, ano das publicações, países, idiomas e áreas do conhecimento.

\section{Principals AUtORES}

O Gráfico 2 apresenta os dez principais autores que publicaram artigos com os temas Strategic Management and Sustainable Tourism no período analisado. 


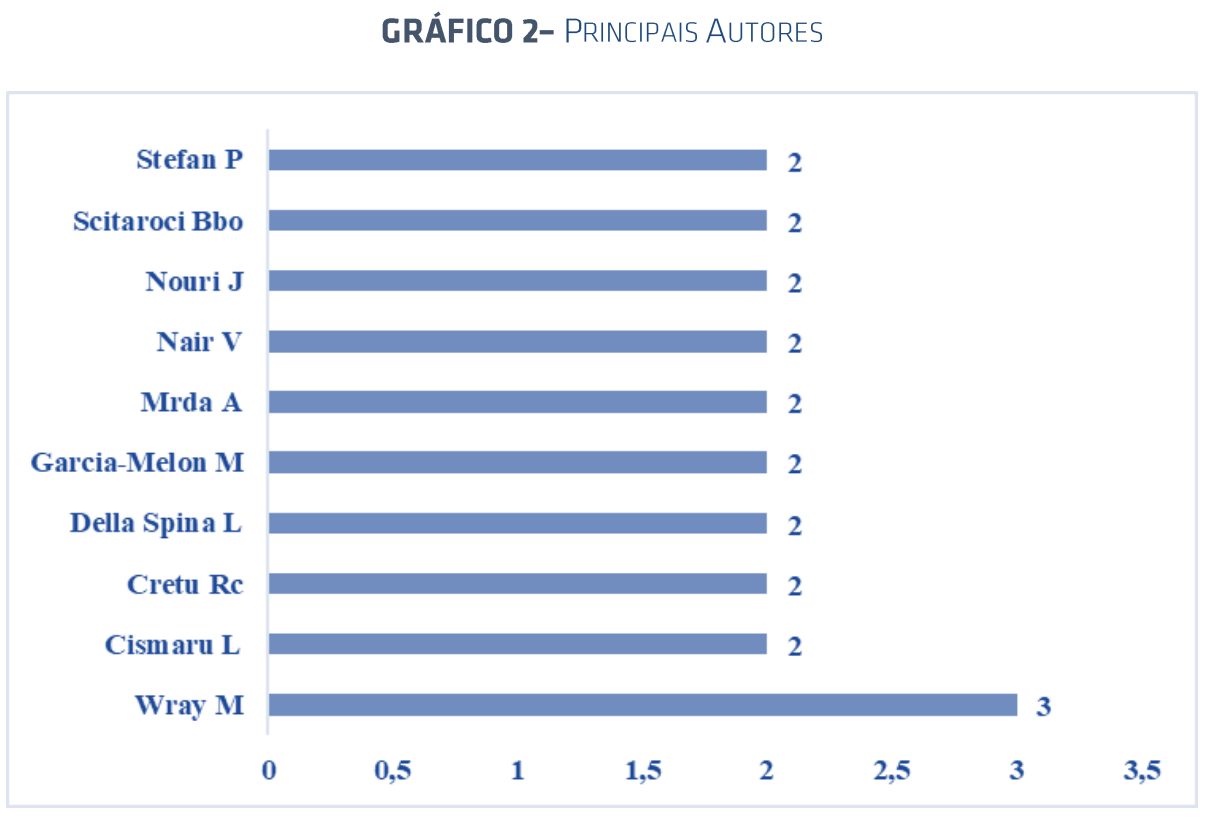

Fonte: Elaborado pelas autoras

Observou-se uma multiplicidade e diversidade quanto à autoria dos trabalhos envolvendo as temáticas analisadas. Um dos autores destaca-se com 3 (três) artigos publicados no período em análise, e os restantes autores publicaram dois artigos no mesmo período. Assim, verifica-se que não existe um investigador que seja um grande expoente quando se analisa as áreas de Strategic Management and Sustainable Tourism simultaneamente.

\section{TITULLO DAS FONTES}

O Gráfico 3 apresenta as principais fontes de publicações e a quantidade de artigos publicados relacionadas com o tema Strategic Management and Sustainable Tourism.

GRÁFICO 3- QUANTIDADE DE ARTIGOS POR FONTE

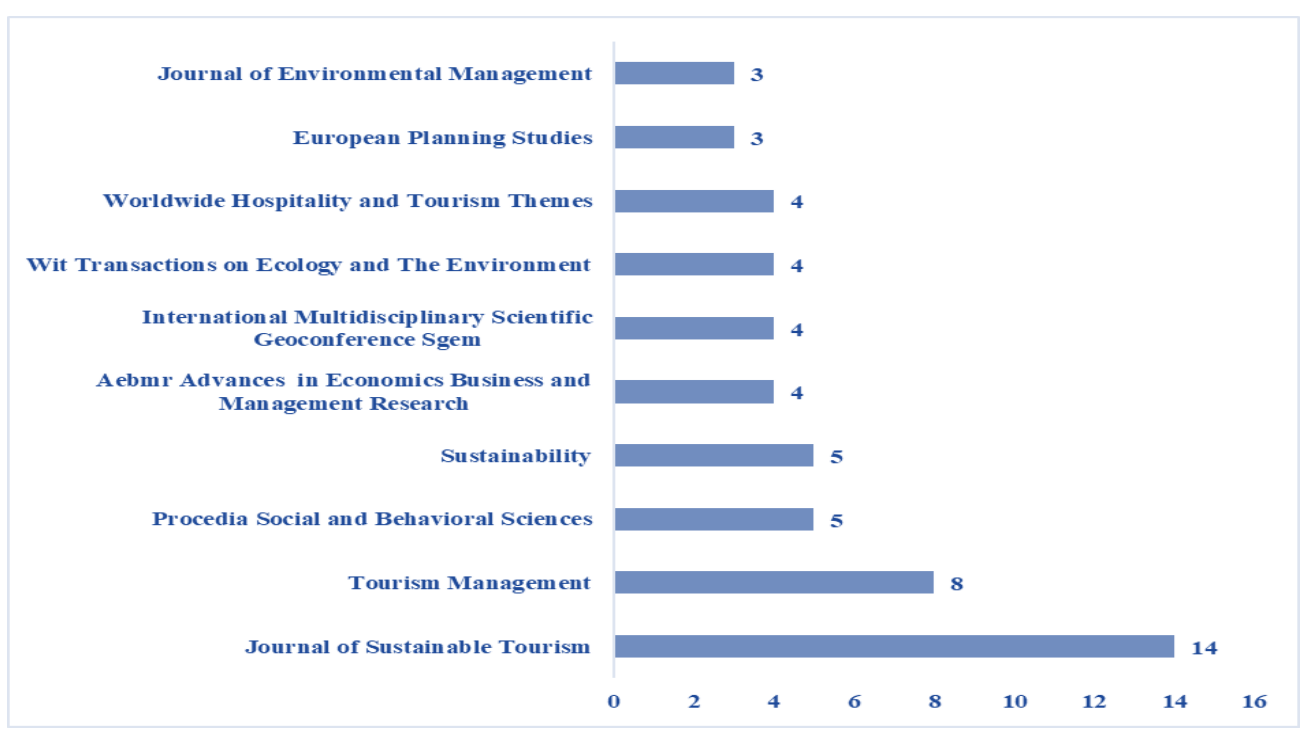

OS, Organizações e Sustentabilidade, 8(1), p. 58-74, Londrina, PR, jan./jun. 2020.

ISSN 2318-9223, http://www.uel.br/revistas/uel/index.php/ros/issue/view/1634/showToc 
Portanto, os jornais que mais publicaram artigos envolvendo o tema foram: Journal of Sustainable Tourism (14), Tourism Management (8) e Procedia Social and Behavioral Sciences (5), Sustainability (5). Parece que os jornais da área são referência de maior aceitação para os artigos destes temas, embora haja também um menor número de artigos em revistas multidisciplinares.

\section{Principais InStituições}

As dez instituições que mais se destacaram e publicaram trabalhos relacionados com o tema, Strategic Management and Sustainable Tourism, estão apresentadas no Gráfico 4.

\section{GRÁFICO 4- PRINCIPAIS INSTITUIÇÕES}

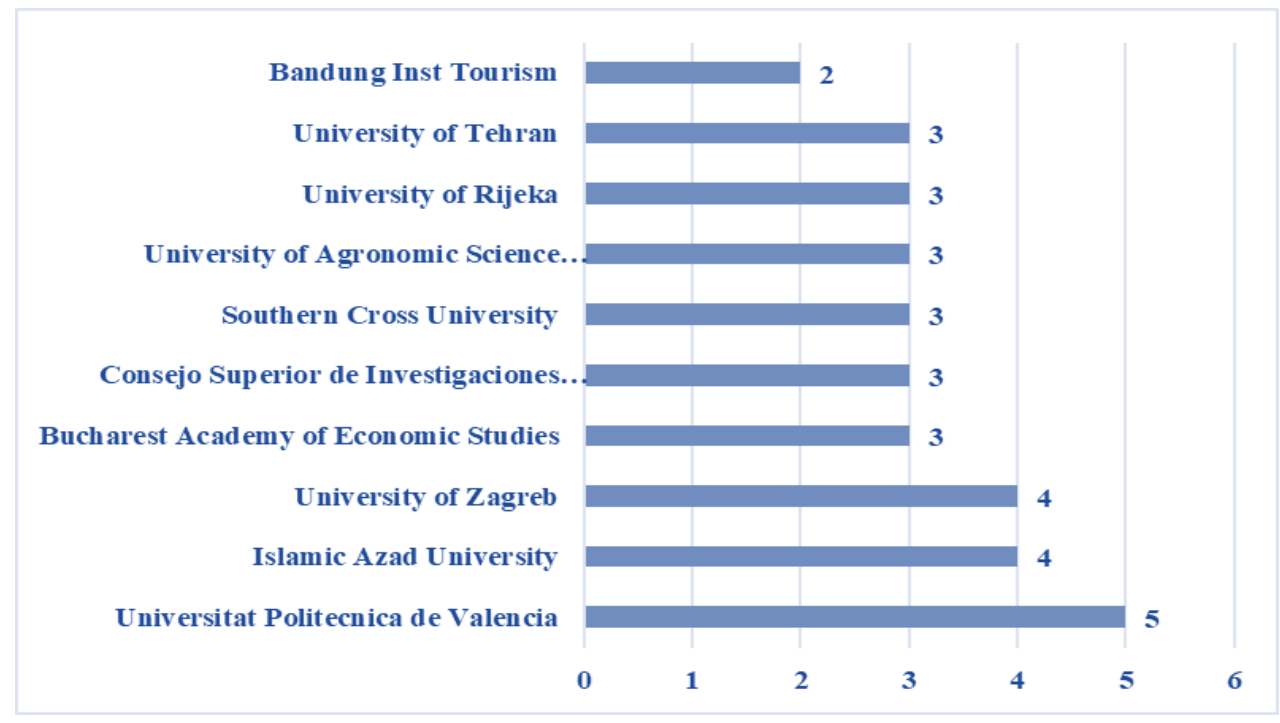

Fonte: Elaborado pelas autoras.

Assim, as instituições que mais publicaram foram: Universitat Politecnica de Valencia em Valência com 5 publicações, a Islamic Azad University no Teerão, e a University of Zagreb na Croácia com 4 publicações cada. Os dados apresentados acima evidenciam a abrangência e importância do tema em instituições de ensino ao redor do mundo.

\section{ANOS de PubLicaÇão}

O Gráfico 5 mostra o número de artigos da Web of Science relacionados com o tema Strategic Management and Sustainable Tourism que foram publicados entre os anos de 2007 e 2017. 


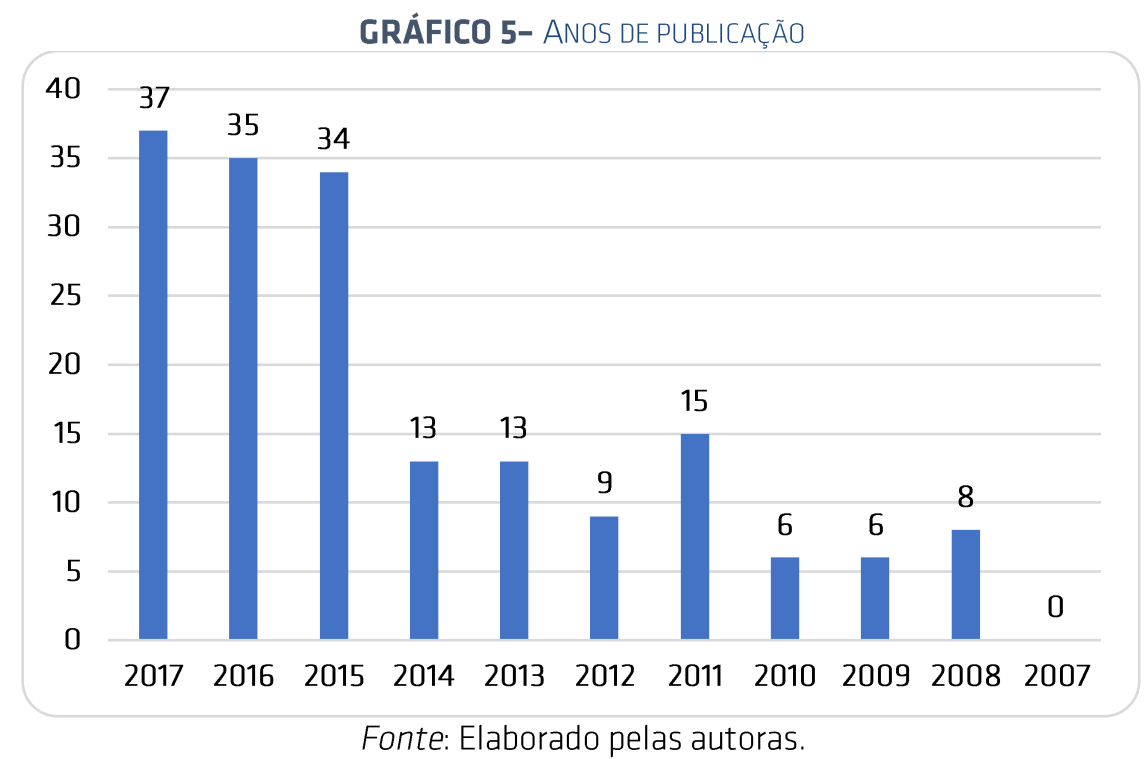

Conforme os dados evidenciados, os anos de 2014 e 2015 houve um crescimento expressivo no número de publicações que. Diante disso, é possível salientar que o interesse e os estudos sobre esses temas tem se ampliado em nivel internacional o que ressalta a importância da temática.

\section{PrincipaIS PaÍSes}

O Gráfico 6 mostra a quantidade de artigos distribuídos pelos principais países.

GRÁFICO 6- QUANTIDADE DE ARTIGOS POR PAÍS

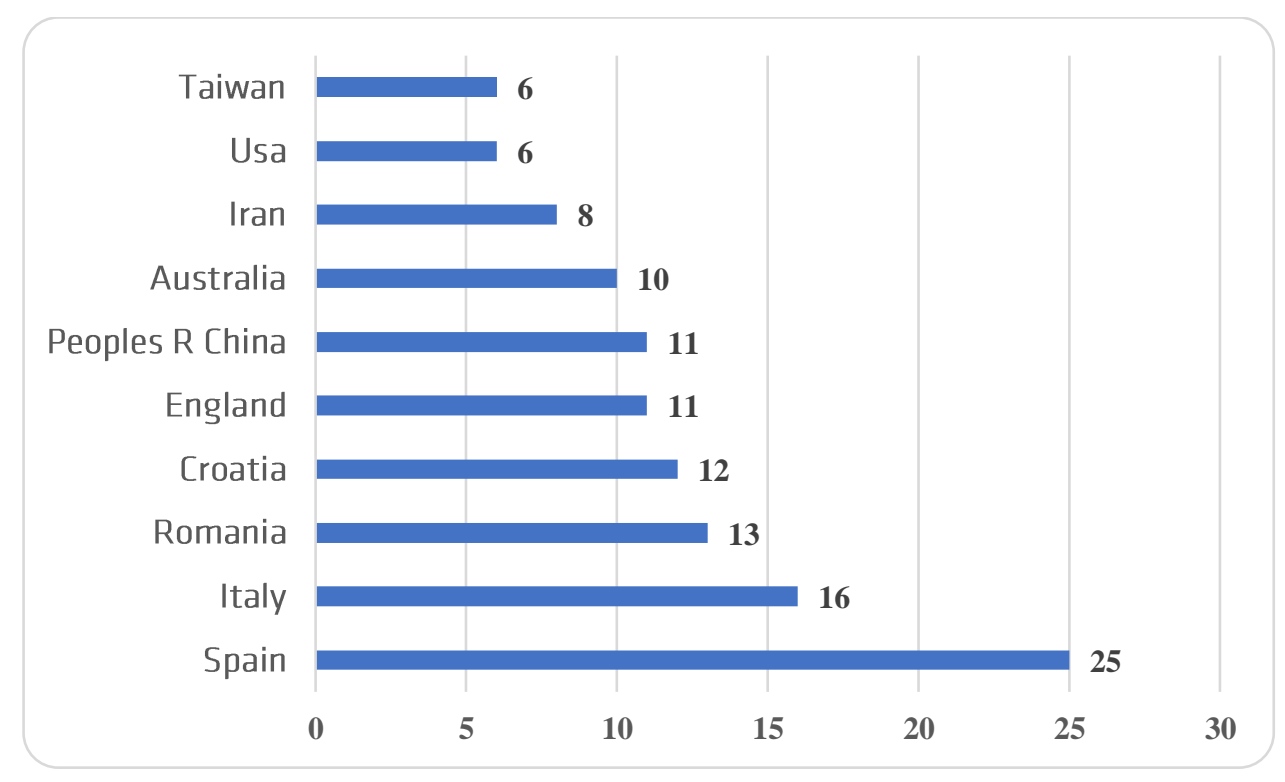

Fonte: Elaborado pelas autoras.

Verificou-se que os quatro países que mais artigos publicaram foram: a Espanha (25), Itália (16), Romênia (13) e Croácia (12). Destaca-se, ainda que, em décimo primeiro lugar temos Portugal com cinco artigos publicados na Web of Science sobre o tema Strategic Management and Sustainable 
Tourism, o que indica que esta temática ainda está pouco trabalhada. No que tange ao Brasil não foram encontrados citações sobre essas temáticas sobre o assunto.

\section{PrincIPAIS IDIOMAS}

No que se refere aos idiomas dos trabalhos publicados na área de estudo estão publicados em inglês (156), conforme se demonstra no Gráfico 7, correspondendo a 88,64\% dos trabalhos.

GRÁFICO 7- QUANTIDADE DE ARTIGOS POR IDIOMA

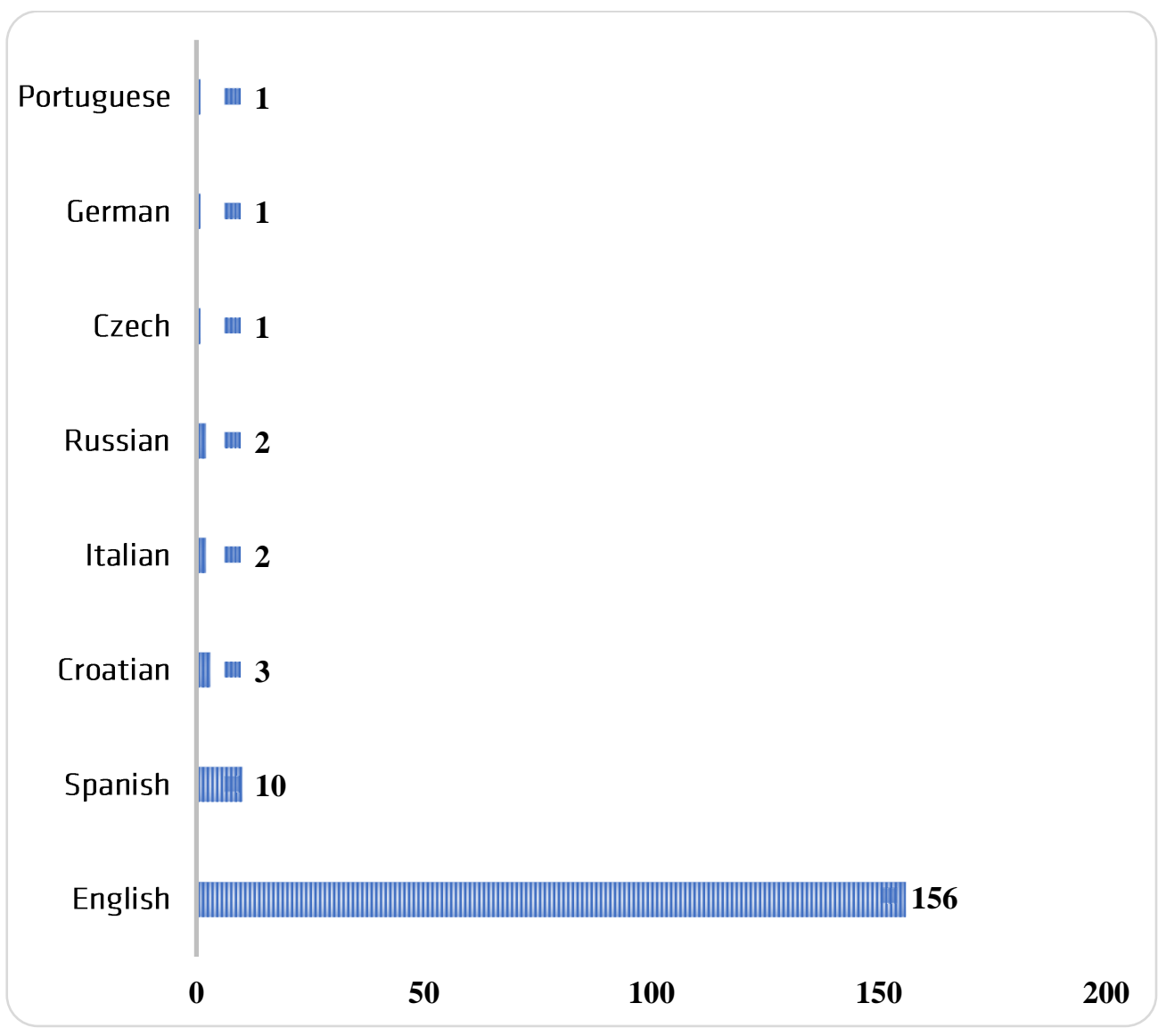

Fonte: Elaborado pelas autoras.

\section{ÁREAS DO CONHECIMENTO}

As áreas do conhecimento com maior número de publicações foram as Social Sciences other topics, com 61, Environmental Sciences Ecologycom 49, Business Economics com 36 e Science Technology other topics com 24, conforme Quadro 1.

\begin{tabular}{|c|c|}
\hline Áreas & $\mathbf{N}^{0}$ Artigos \\
\hline Social Sciences other Topics & 69 \\
\hline Environmental Sciences Ecology & 50 \\
\hline Business Economics & 46 \\
\hline Science Technology other Topics & 26 \\
\hline
\end{tabular}

OS, Organizações e Sustentabilidade, 8(1), p. 58-74, Londrina, PR, jan./jun. 2020.

ISSN 2318-9223, http://www.uel.br/revistas/uel/index.php/ros/issue/view/1634/showToc 


\begin{tabular}{|l|l|}
\hline Geology & 10 \\
\hline Public Administration & 9 \\
\hline Geography & 8 \\
\hline Water Resources & 7 \\
\hline Agriculture & 5 \\
\hline Engineering & 5 \\
\hline
\end{tabular}

Fonte: Elaborado pelas autoras.

O total dos artigos é superior ao número de publicações (176), porque um artigo pode estar vinculado a mais do que uma área.

\section{ARTIGOS MAIS CITADOS NO PERIOdO DE 2007 A 2017}

No período de 2007 a 2017, identificaram-se 869 citações, dos 17 (dezessete) artigos mais citados sobre Strategic Management and Sustainable Tourism realizada na base de dados Web of Science, apresentados no Quadro 2.

QUADRO 2 - RELAÇÃO DAS 10 PUBLICAÇÕES MAIS CITADAS NO PERÍODO (2007 A 2017)

\begin{tabular}{|c|c|c|c|c|}
\hline $\begin{array}{l}\text { Number of } \\
\text { Quotations } \\
2010-2017 \\
\end{array}$ & Title & Author & Journal & Year \\
\hline 167 & $\begin{array}{l}\text { Destination and enterprise } \\
\text { management for a tourism } \\
\text { future }\end{array}$ & $\begin{array}{l}\text { Dwyer, } \quad \text { Larry; } \\
\text { Edwards, Deborah; } \\
\text { Mistilis, Nina; Roman, } \\
\text { Carolina; Scott, Noel }\end{array}$ & $\begin{array}{l}\text { Tourism } \\
\text { Management }\end{array}$ & 2009 \\
\hline 123 & $\begin{array}{l}\text { Integrated rural } \\
\text { Concepts and practice }\end{array}$ & $\begin{array}{l}\text { Cawley, Mary; Gillmor, } \\
\text { Desmond A. }\end{array}$ & $\begin{array}{l}\text { Annals } \quad \text { of } \\
\text { Tourism } \\
\text { Research }\end{array}$ & 2008 \\
\hline 105 & $\begin{array}{lr}\text { Implementing } & \text { sustainable } \\
\text { tourism: A multi-stakeholder } \\
\text { involvement } \\
\text { framework }\end{array}$ & $\begin{array}{l}\text { Waligo, Victoria M.; } \\
\text { Clarke, } \quad \text { Jackie; } \\
\text { Hawkins, Rebecca }\end{array}$ & $\begin{array}{l}\text { Tourism } \\
\text { Management }\end{array}$ & 2013 \\
\hline 54 & $\begin{array}{l}\text { Exploring the travel behaviors of } \\
\text { inbound tourists to Hong Kong } \\
\text { using geotagged photos }\end{array}$ & $\begin{array}{l}\text { Huy Quan Vu; Li, } \\
\text { Gang; Law, Rob; Ye, } \\
\text { Ben Haobin }\end{array}$ & $\begin{array}{l}\text { Tourism } \\
\text { Management }\end{array}$ & 2015 \\
\hline 49 & $\begin{array}{l}\text { Hotel water consumption at a } \\
\text { seasonal mass tourist } \\
\text { destination. The case of the } \\
\text { island of Mallorca }\end{array}$ & $\begin{array}{lr}\text { Deya } & \text { Tortella, } \\
\text { Bartolome; } & \text { Tirado, } \\
\text { Dolores } & \end{array}$ & $\begin{array}{l}\text { Journal Of } \\
\text { Environmental } \\
\text { Management }\end{array}$ & 2011 \\
\hline 47 & $\begin{array}{l}\text { Building dynamic capabilities } \\
\text { through knowledge resources }\end{array}$ & $\begin{array}{l}\text { Nieves, Julia; Haller, } \\
\text { Sabine }\end{array}$ & $\begin{array}{l}\text { Tourism } \\
\text { Management }\end{array}$ & 2014 \\
\hline 38 & $\begin{array}{l}\text { Event tourism governance and } \\
\text { the public sphere }\end{array}$ & $\begin{array}{l}\text { Dredge, Dianne; } \\
\text { Whitford, Michelle }\end{array}$ & $\begin{array}{l}\text { Journal Of } \\
\text { Sustainable } \\
\text { Tourism }\end{array}$ & 2011 \\
\hline 36 & $\begin{array}{l}\text { Multiple Carrying Capacities } \\
\text { from a management-oriented } \\
\text { perspective to operationalize } \\
\text { sustainable tourism in protected } \\
\text { areas }\end{array}$ & $\begin{array}{lr}\text { Salerno, } & \text { Franco; } \\
\text { Viviano, } & \text { Gaetano; } \\
\text { Manfredi, Emanuela } \\
\text { C.; Caroli, } \quad \text { Paolo; } \\
\text { Thakuri, } \quad \text { Sudeep; } \\
\text { Tartari, Gianni }\end{array}$ & $\begin{array}{l}\text { Journal Of } \\
\text { Environmental } \\
\text { Management }\end{array}$ & 2013 \\
\hline 35 & $\begin{array}{l}\text { The cultural and environmental } \\
\text { resources for sustainable } \\
\text { development of rural areas in } \\
\text { economically disadvantaged } \\
\text { contexts. Economic-appraisals }\end{array}$ & $\begin{array}{l}\text { Calabro, Francesco; } \\
\text { Della Spina, Lucia }\end{array}$ & $\begin{array}{l}\text { Sustainable } \\
\text { Development of } \\
\text { Industry And } \\
\text { Economy, Pts } 1 \\
\text { And } 2\end{array}$ & 2014 \\
\hline
\end{tabular}

OS, Organizações e Sustentabilidade, 8(1), p. 58-74, Londrina, PR, jan./jun. 2020.

ISSN 2318-9223, http://www.uel.br/revistas/uel/index.php/ros/issue/view/1634/showToc 


\begin{tabular}{|c|c|c|c|c|}
\hline & $\begin{array}{l}\text { issues of a model of } \\
\text { management for } \\
\text { valorisation of public assets }\end{array}$ & & & \\
\hline 34 & $\begin{array}{l}\text { Critical review of strategic } \\
\text { planning research in hospitality } \\
\text { and tourism }\end{array}$ & $\begin{array}{l}\text { Phillips, } \\
\text { Moutinho, Luiz }\end{array}$ & $\begin{array}{l}\text { Annals } \quad \text { Of } \\
\text { Tourism } \\
\text { Research }\end{array}$ & 2014 \\
\hline 34 & $\begin{array}{ll}\text { A combined ANP-delphi } \\
\text { approach to evaluate } \\
\text { sustainable tourism }\end{array}$ & \begin{tabular}{ll}
\multicolumn{2}{l}{ Carcia-Melon, } \\
Monica; $\quad$ Gomez- \\
Navarro, Tomas; \\
Acuna-Dutra, Silvia \\
\end{tabular} & $\begin{array}{l}\text { Environmental } \\
\text { Impact } \\
\text { Assessment } \\
\text { Review }\end{array}$ & 2012 \\
\hline 32 & $\begin{array}{l}\text { A Framework for Sustainable } \\
\text { Heritage Management: A Study } \\
\text { of UK Industrial Heritage Sites }\end{array}$ & Landorf, Chris & $\begin{array}{l}\text { International } \\
\text { Journal of } \\
\text { Heritage } \\
\text { Studies }\end{array}$ & 2009 \\
\hline 28 & $\begin{array}{l}\text { Exploring the Gap between } \\
\text { Ecosystem Service Research and } \\
\text { Management in Development } \\
\text { Planning }\end{array}$ & $\begin{array}{l}\text { Sitas, } \quad \text { Nadia; } \\
\text { Prozesky, Heidi E.; } \\
\text { Esler, Karen J.; Reyers, } \\
\text { Belinda }\end{array}$ & Sustainability & 2014 \\
\hline 27 & $\begin{array}{l}\text { Can tourism be part of the } \\
\text { decarbonized global economy? } \\
\text { The costs and risks of alternate } \\
\text { carbon reduction policy } \\
\text { pathways }\end{array}$ & $\begin{array}{l}\text { Scott, Daniel; } \\
\text { Gossling, Stefan; Hall, } \\
\text { C. Michael; Peeters, } \\
\text { Paul }\end{array}$ & $\begin{array}{ll}\text { Journal } & \text { Of } \\
\text { Sustainable } & \\
\text { Tourism } & \end{array}$ & 2016 \\
\hline 26 & $\begin{array}{l}\text { Strategic organizational drivers } \\
\text { of corporate environmental } \\
\text { responsibility in the Caribbean } \\
\text { hotel industry }\end{array}$ & Shah, Kalim U. & Policy Sciences & 2011 \\
\hline 26 & $\begin{array}{l}\text { Conceptualising a contemporary } \\
\text { marketing mix for sustainable } \\
\text { tourism }\end{array}$ & $\begin{array}{l}\text { Pomering, Alan; } \\
\text { Noble, Gary; Johnson, } \\
\text { Lester W. }\end{array}$ & $\begin{array}{l}\text { Journal Of } \\
\text { Sustainable } \\
\text { Tourism }\end{array}$ & 2011 \\
\hline 25 & $\begin{array}{l}\text { Using indicators to assess } \\
\text { sustainable } \\
\text { development: a review }\end{array}$ & $\begin{array}{l}\text { Torres-Delgado, } \\
\text { Anna; Saarinen, } \\
\text { Jarkko }\end{array}$ & $\begin{array}{l}\text { Tourism } \\
\text { Geographies }\end{array}$ & 2014 \\
\hline 23 & $\begin{array}{l}\text { Environmental supply chain } \\
\text { management in tourism: The } \\
\text { case of large tour operators }\end{array}$ & Budeanu, Adriana & $\begin{array}{ll}\text { Journal } & \text { Of } \\
\text { Cleaner } & \\
\text { Production } & \\
\end{array}$ & 2009 \\
\hline 22 & $\begin{array}{lrr}\text { BEST } & \text { PRACTICE } & \text { MARKETING } \\
\text { FOR } & \text { REGIONAL } & \text { TOURISM } \\
\text { DESTINATIONS } & \\
\end{array}$ & $\begin{array}{l}\text { Cox, Carmen; Wray, } \\
\text { Meredith }\end{array}$ & $\begin{array}{l}\text { Journal Of Travel } \\
\text { \& } \quad \text { Tourism } \\
\text { Marketing }\end{array}$ & 2011 \\
\hline 22 & $\begin{array}{l}\text { Evaluation of ecotourism } \\
\text { potential in the northern } \\
\text { coastline of the Persian Gulf }\end{array}$ & $\begin{array}{lr}\text { Nouri, } & \text { Jafar; } \\
\text { Danehkar, Afshin; } \\
\text { Sharifipour, Rozita }\end{array}$ & $\begin{array}{l}\text { Environmental } \\
\text { Geology }\end{array}$ & 2008 \\
\hline 21 & $\begin{array}{l}\text { Networking for sustainable } \\
\text { tourism - towards a research } \\
\text { agenda }\end{array}$ & Albrecht, Julia N. & $\begin{array}{l}\text { Journal } \\
\text { Sustainable } \\
\text { Tourism }\end{array}$ & 2013 \\
\hline
\end{tabular}

Fonte: Elaborado pelas autoras.

Comparando o quadro 2 com o Gráfico 2, foi possivel constatar que 4 (quatro) dos dez autores que mais publicaram sobre o tema no período entre 2007 e 2017 estão entre os 21(vinte e um) autores mais citados. Temos, por exemplo, os autores Lucia Della Spina e Francesco Calabro com 35 citações, que escreveram o artigo com o título "The cultural and environmental resources for sustainable development of rural areas in economically disadvantaged contexts. Economic-appraisals issues of a model of management for the valorisation of public assets»; Monica Garcia-Melon coautora com Tomas Gomez-Navarro e Silvia Acuna-Dutra com 34 citações escreveram o artigo com 0 título « $A$ combined ANP-delphi approach to evaluate sustainable tourism». Temos, com 22 citações os artigos «Evaluation of ecotourism potential in the northern coastline of the Persian Gulf» escrito por Jafar Nouri, Afshin Danehkar e Rozita Sharifipour; outro com 22 foi o escrito por Meredith Wray coautora

0S, Organizações e Sustentabilidade, 8(1), p. 58-74, Londrina, PR, jan./jun. 2020. 
com Carmen Cox, com o título «Best practice marketing for regional tourism destinations» na Web of Science naquele período.

\section{CONSIDERAÇÕES FINAIS}

Este estudo teve como objetivo apresentar o Estado da arte e procurar discutir as interfaces e articulações entre os constructos Gestão Estratégica e Turismo Sustentável. Para concretizar o objetivo do estudo realizou-se uma pesquisa bibliométrica utilizando a base de dados Web of Science, cuja amostra final resultou na análise de 176 artigos.

O tema foi considerado interdisciplinares, devido aos artigos estarem relacionados a mais do que uma área do conhecimento. Quanto às perspetivas de pesquisa, mostra que os interesses e focos de investigação sobre o tema Gestão Estratégica e Turismo Sustentável estão em crescimento para o conhecimento científico. Com isso, foi possivel mapear e analisar o cenário de produção científica relacionada ao tema Gestão Estratégica e Turismo Sustentável.

Em relação ao veículo de divulgação, verificou-se que o Journal of Sustainable Tourismapresentou o maior número de publicações (14), seguido do Tourism Management (8) e dos Procedia Social and Behavioral Sciences e Sustainabilitycom 5 publicações cada.

A concentração das publicações foi em países como Espanha, Itália, Roménia e Croácia. Portugal ocupa a décima primeira posição dos 25 países que mais publicaram sobre o tema e o Brasil não consta da listagem.

Quanto aos autores que mais publicaram nesse tema, observou-se uma multiplicidade e diversidade quanto à autoria dos trabalhos. Percebeu-se, portanto, que quatro dos dez autores que mais publicaram sobre o tema constam da listagem dos 21 (vinte e um) autores mais citados: Meredith Wray com 3 publicações, Lucia Della Spina (2), Monica Garcia-Melon (2) e Jafar Nouri (2). As instituições que mais se destacaram no que se refere as publicações relacionadas com Strategic Management and Sustainable Tourism, foram: Universitat Politecnica de Valencia, Islamic Azad Universitye University of Zagreb.

A contribuição desse estudo para os estudos em Gestão deve-se aos indicadores resultantes quanto as instituições de pesquisa e aos journals que mais se destacam na produção do conhecimento sobre o tema a nivel internacional.

\section{LIMITAÇÕES E FUTURAS LINHAS DE INVESTIGAÇÃO}

A principal limitação deste estudo refere-se ao fato das informações apresentadas estarem limitadas aos artigos encontrados na base Web of Science.

Para estudos futuros sugere-se a ampliação de pesquisa, sobre o tema Gestão Estratégica e Turismo Sustentável, em outras bases de dados, bem como em outros eventos científicos nacionais e internacionais, de maneira a se obter outros resultados sobre o perfil bibliométrico das publicações relacionadas com o tema.

Constatou-se que não havia nenhum investigador português, nem brasileiro, na listagem dos dez autores que mais publicaram sobre o tema, o que reforça a ideia de que este tema precisa de ser mais estudado.

\section{REFERÊNCIAS}

Almeida, M.I.R. (2010). Manual de Planejamento Estratégico - Desenvolvimento de um Plano Estratégico com a Utilização de Planilhas Excel. 3. ed. São Paulo: Atlas. 
Araújo, C.A. (2006). Bibliometria: evolução histórica e questões atuais. Em Questão, Enero Junio, 12(1), 11-32.

Araújo, WA.; Temoteo, J.A.G.; Andrade, M.O.; Trevizan, S.P. (2017). Local development, tourism and traditional populations: conceptual elements and notes for reflection. Interações, Campo Grande, MS, $(18,4), 5-18$.

Balsadi, O.V. (2001). Mudanças no meio rural e desafios para o desenvolvimento sustentável, São Paulo Perspec., 15(1), 155-165.

Butler, R. (2004). Issues in applying carrying capacity concepts: examples from United Kigdom. In: Coccossis, H. Mexa, A. Planning and management for tourism growth isbecoming essential in the context of sustainable development. (pp 135-150).1. ed.

Dias, R. (2006). Gestão Ambiental: Responsabilidade social e sustentabilidade. São Paulo, Atlas.

Diniz, P. C. de O. C.; Giarola, E.; Balbino, R. F.; Ferreira, R. N.; Nazareth, L. G. C. (2013). A estratégia empresarial e o marketing estratégico como forma de fortalecimento da doutrina cooperativista: uma pesquisa aplicada sob o enfoque da teoria dos jogos. Revista de Administração Faces, Belo Horizonte, 12(3), 64-81.

Farjoun, M. (2002). Towards an organic perspective on strategy. Strategic Management Journal, 23(7), 561-594.

Freire, A. (2008). Estratégia - Sucesso em Portugal. 11ª Ed. Lisboa / São Paulo: Verbo.

Hall, J.; Matos, S. (2010). "Incorporating impoverished communities in sustainable supply chains", International Journal of Physical Distribution \& Logistics Management, 40(1/2), 124-147.

Hamel, G. (2001). Competindo pelo futuro: estratégias inovadoras para obter o controle do seu setor e criar os mercados de amanhã. 12. ed. Rio de Janeiro: Campus.

Hitt, M. A.; Ireland, R. D.; Hoskisson, R. E. (2011). Administração estratégica: competitividade e globalização. 2 ed. São Paulo: Cengage Learning, 2011.

Kneipp, J. M. Gestão estratégica da inovação sustentável e sua relação com o modelo de negócio e o desempenho empresarial. 2016. Tese (Doutorado). Centro de Pós-Graduação em Administração, Universidade Federal de Santa Maria - UFSM, Rio Grande do Sul, 2016.

Leví, M. (2012). O Turismo e Desenvolvimento Sustentável: Contributos do Turismo de Natureza no Desenvolvimento do Parque Nacional da Gorongosa. 132f. Trabalho de conclusão de curso (Dissertação) - Faculdade de Ciências Sociais e Humanas, Universidade Lusófona de Humanidades e Tecnologias, Lisboa.

Lima, W.C.; Luz, T.R. (2012). A estratégia como ferramenta de gestão e desempenho organizacional - estudo de caso em uma microempresa - Araxá-MG. Evidência, Araxá, 8(8) 41-54.

Liu, W., Vogt, A.C., Luo, J., He, G., Frank, A.K. \& Liu, J. (2012). Drivers and Socioeconomic Impacts of Tourism Participation in Protected Areas. Plos One, 7(4), 1-14.

Lozada, L. (2017). Serviços ecossistêmicos e interações com uma comunidade afrodescendente no Pacífico colombiano: dos riscos à proteção da biodiversidade. Dissertação (Mestrado em Desenvolvimento e Meio Ambiente) - Universidade Federal da Paraíba, João Pessoa, PB.

Martins, P.S., Filho, E.E., Nagano, M.S. (2015). Gestão ambiental e estratégia empresarial em pequenas e médias empresas: um estudo comparativo de casos. Engenharia Sanitária e Ambiental, São Carlos, 20(29), 225-234.

Medeiros, L. \& Moraes, P. (2013). Turismo e sustentabilidade ambiental: referências para o desenvolvimento de um turismo sustentável. Revista Meio Ambiente e Sustentabilidade, 3(2). 
Mintzberg, H., Lampel, J., Quinn, J.B., Ghoshal S. (2006). O processo da estratégia: conceitos, contextos e casos selecionados. 4 ed. Porto Alegre: Bookman, 496.

Motta, P.R. (1995). A ciência e a arte de ser dirigente. 6. ed. Rio de Janeiro: Record.

Nicolau, I. (2001). 0 conceito de estratégia. Recuperado de: <htttp://www.iscte.pt/Estrategia/conceito\%20estrategia.pdf>. Acesso em: 20 out.2017.

Okubo, Y. (1997). Bibliometric Indicators and Analysis of Research Systems: Methods and Examples, OECD Science, Technology and Industry Working Papers, OECD Publishing, Paris.

Oliveira, M.H. (2013). Definição e Execução de uma Estratégia Empresarial. Acesso em: 19 out. 2017. Organização Mundial do Turismo (OMT). (2001). Introdução ao turismo. São Paulo: Roca. 371.

Organização Mundial do Turismo (OMT) (2014). Crónicas del turismo: el desarrollo comunitario sobre el terreno.

Parnell, J.A. (2010). Strategic clarity, business strategy and performance. Journal of Strategy and Management, 3(4), 304-324.

Pires, P. (2002). Dimensões do Ecoturismo. São Paulo: Editora SENAC.

Pontes, A.S.M. (2012). Análise do tema gestão estratégica nas pequenas empresas prestadoras de serviços: uma revisão bibliográfica. Gestão e Tecnologia, Florianópolis, 2(2), 26-32.

Porter, M. (2005). Estratégia competitiva: Técnicas para a análise de indústrias e da concorrência. Rio de Janeiro: Campus.

Rocha, B.M., Zouain, M.D. (2015). Percepção socioambiental: a visão de turistas e gestores de hotéis sobre os impactos da poluição das praias no turismo do Rio de Janeiro. Revista Brasileira de Pesquisa em Turismo. São Paulo, 9(2), 360-377.

Rostaing, H. (1997). La bibliométrie et sés techniques. Toulouse: Sciences de la Société; Marseille: Centre de Recherche Rétrospective de Marseille.

Savitz. A., Weber. K. (2007). A Empresa Sustentável: O verdadeiro sucesso é o lucro com responsabilidade social e ambiental. São Paulo, Campus/Elsevier.

Silva, M.R. (2004). Análise bibliométrica da produção científica docente do programa de pósgraduação em educação especial/UFSCar. Dissertação (Mestrado em Educação Especial) Universidade Federal de São Carlos, São Carlos, Brasil.

UNESCO. (2017). Organização das Nações Unidas para a Educação, a Ciência e a Cultura. A UNESCO e 0 ano internacional do turismo sustentável. Recuperado de: <http://www.unesco.org/new/pt/brasilia/about-this-office/prizes-and-celebrations/2017international-year-of-sustainable-tourism/ >. Acesso em: 01/10/2017.

Wakeford, J.J., Gebreeyesus, M., Ginbo, T., Yimer, K., Manzambi, O., Okereke, C., Black, M., Mulugetta, Y. Innovation for green industrialisation: An empirical assessment of innovation in Ethiopia's cement, leather and textile sectors, Journal of Cleaner Production. 166,p. 503-511,2017.

World Travel \& Tourism Council (WTTC). (2012). The indirect impact of tourism: an economic analysis. Recuperado de: < http://cf.cdn.unwto.org/sites/all/files/111020-rapport_vellas_en.pdf >. Acesso em: 01/10 /2017. 